$06,11,04$

\title{
Фазовые диаграммы твердых растворов сегнетоэлектриков-релаксоров по данным диэлектрической спектроскопии
}

\author{
(C) М.В. Таланов, Л.А. Резниченко
}

Научно-исследовательский институт физики Южного федерального университета, Ростов-на-Дону, Россия

E-mail: mvtalanov@sfedu.ru

Исследованы диэлектрические спектры керамики состава $\mathrm{Pb}_{(1-z)} \mathrm{Ba}_{z}\left(\mathrm{Mg}_{1 / 3} \mathrm{Nb}_{2 / 3}\right)_{m}\left(\mathrm{Zn}_{1 / 3} \mathrm{Nb}_{2 / 3}\right)_{y}\left(\mathrm{Ni}_{1 / 3} \mathrm{Nb}_{2 / 3}\right)_{n} \mathrm{Ti}_{x} \mathrm{O}_{3}$ $(x=0.25-0.4, y=0.1130-0.0842, m=0.4844-0.1298, n=0.1266-0.4726, z=0-0.15)$ с замещением как в $A$-, так и в $B$-кристаллографических позициях структуры перовскита. При увеличении концентрации титаната свинца, так же как и при уменьшении концентрации бария, в системе наблюдается переход из релаксорного в нормальное сегнетоэлектрическое состояние. На основании экспериментальных результатов построены $x-T$ и $z-T$ фазовые диаграммы, которые, несмотря на различные кристаллохимические мотивы возникновения релаксорного состояния в исследуемых твердых растворах, демонстрируют очевидное сходство. Обнаружено исчезновение температурного гистерезиса при переходе в релаксорное состояние в обоих случаях, что позволило нам сделать предположение о существовании трикритических точек на соответствующих фазовых диаграммах.

Работа выполнена при финансовой поддержке РФФИ в рамках научного проекта № 16-32-60025 мол_а_дк и государственного задания Минобрнауки России: проекты № 3.6371.2017/8.9, 3.6439.2017/8.9.

DOI: 10.21883/FTT.2018.03.45540.10D

\section{1. Введение}

Сегнетоэлектрики-релаксоры (СЭР) - класс неупорядоченных на наноразмерном уровне объектов, твердые растворы которых вблизи морфотропных фазовых границ (МФГ), разделяющих тетрагональную и ромбоэдрическую области $x$ - $T$ фазовых диаграмм, демонстрируют рекордные значения пьезоэлектрических откликов [1]. Это делает возможным их использование в широком диапазоне устройств: актюаторах, сенсорах, преобразователях, устройствах накопления энергии и др. [2,3]. Считается, что при охлаждении СЭР до температуры Даколя-Бернса $\left(T_{d}\right)$ в параэлектрической матрице появляются динамические полярные области нанометрового масштаба, а сам кристалл переходит в эргодическое релаксорное состояние [4]. Дальнейшее охлаждение приводит к росту количества и объема полярных нанообластей, которые становятся статическими при температуре $T^{*}$, и заморозке их динамики ниже температуры Фогеля-Фулчера $\left(T_{\mathrm{VF}}\right)$ и к переходу в неэргодическое релаксорное состояние [5-9]. Под влиянием химического модифицирования, давления и/или электрического поля в твердых растворах (ТР) СЭР возможны обратимые и необратимые переходы между эргодическим релаксорным, неэргодическим релаксорным и сегнетоэлектрическим состояниями.

По-видимому, основной причиной такого поведения СЭР является неупорядоченное распределение различных ионов в узлах одной кристаллографической позиции, что приводит к появлению случайных электрических полей, ответственных за необычное проявление макроскопических откликов, в частности диэлек- трических [10,11]. Известны многие СЭР со структурой типа перовскита с гетеро/изовалентным замещением катионов как в $A-\left(\mathrm{Pb}_{1-x} \mathrm{La}_{x}\left(\mathrm{Zr}_{0.65} \mathrm{Ti}_{0.35}\right)_{1-x / 4} \mathrm{O}_{3}\right.$, $\left.(1-x) \mathrm{Na}_{1 / 2} \mathrm{Bi}_{1 / 2} \mathrm{TiO}_{3}-x \mathrm{BaTiO}_{3}\right)$, так и $B-\left(\mathrm{PbMg}_{1 / 3} \mathrm{Nb}_{2 / 3} \mathrm{O}_{3}\right.$, $\left.\mathrm{BaTi}_{1-x} \mathrm{Zr}_{x} \mathrm{O}_{3}, \mathrm{PbSc}_{1 / 2} \mathrm{Ta}_{1 / 2} \mathrm{O}_{3}\right)$ кристаллографических позициях. Однако в большинстве известных бинарных систем с участием СЭР и классических сегнетоэлектриков $\left(\mathrm{PbTiO}_{3}, \mathrm{BaTiO}_{3}\right.$ и др.) МФГ формируются в весьма узком диапазоне концентраций, что усложняет их исследование, а также затрудняет выбор составов ТР с необходимым набором функциональных параметров. Одним из решений этой проблемы является конструирование многокомпонентных систем ТР, что существенно повышает вариативность составов функциональных материалов за счет увеличения мерности морфотропной области и расширения диапазона ее существования. Данное направление развивается в пьезоэлектрическом материаловедении с 70-х годов XX века, и к настоящему моменту было синтезировано и исследовано значительное количество систем ТР с участием СЭР [12].

Целью данной работы стало установление границ существования различных фазовых состояний ТР СЭР с замещением как в $A$-, так и в $B$-кристаллографических позициях перовскитовой структуры на основе одной из многокомпонентных систем и выявление общих закономерностей проявления диэлектрических свойств в них.

\section{2. Объекты, методы получения и исследования образцов}

В качестве объектов исследования нами были выбраны ТP многокомпонентной системы 
$\mathrm{Pb}_{(1-z)} \mathrm{Ba}_{z}\left(\mathrm{Mg}_{1 / 3} \mathrm{Nb}_{2 / 3}\right)_{m}\left(\mathrm{Zn}_{1 / 3} \mathrm{Nb}_{2 / 3}\right)_{y}\left(\mathrm{Ni}_{1 / 3} \mathrm{Nb}_{2 / 3}\right)_{n} \mathrm{Ti}_{x} \mathrm{O}_{3}$ $(x=0.25-0.4, y=0.1130-0.0842, m=0.4844-0.1298$, $n=0.1266-0.4726, z=0-0.15)$. На основании предварительных экспериментальных результатов [13-17] выбраны два разреза системы, составы которых, при увеличении концентрации титаната свинца $(x)$ и уменьшении концентрации бария $(z)$, демонстрируют одинаковую последовательность структурных фазовых переходов (ФП) из кубической фазы в тетрагональную через МФГ (при $x \sim 0.30$ и $z \sim 0.05$ ). В первом разрезе химический состав изменяется только в $B$-подрешетке, а во втором - только в $A$-подрешетке. Выбранные разрезы пересекаются друг с другом в составе с $x=0.30$ и $z=0.05$.

Изучаемые ТР получены путем твердофазного синтеза с использованием колумбитного метода [18]. Спекание проводилось при температуре $T_{\text {сп. }}=1180-1220^{\circ} \mathrm{C}$. Экспериментальные образцы представляли собой диски диаметром $10 \mathrm{~mm}$ и толщиной $1 \mathrm{~mm}$ с нанесенными (путем двукратного вжигания) на плоские торцевые поверхности серебросодержащими электродами.

Диэлектрические измерения были выполнены с помощью стенда, включающего LCR-meter Agilent E4980A в интервале температур $298-873 \mathrm{~K}$ на частотах измерительного электрического поля $(f) 0.1-1000 \mathrm{kHz}$. Экспериментальные результаты получены с использованием оборудования Центра коллективного пользования „Электромагнитные, электромеханические и тепловые свойства твердых тел“ НИИ физики Южного федерального университета.

\section{3. Экспериментальные результаты и обсуждение}

На рис. 1, $a$ представлены наиболее характерные температурные зависимости величины относительной диэлектрической проницаемости $\left(\varepsilon / \varepsilon_{0}\right)$, измеренной при различных значениях $f$ при охлаждении образцов с $x=0.250,0.325$ и 0.375 . Кривые $\varepsilon / \varepsilon_{0}(T)$ образцов с $x=0.250$ демонстрируют поведение, свойственное СЭР: размытый максимум $\varepsilon / \varepsilon_{0}$, положение которого смещается в сторону высоких температур при возрастании $f$. Увеличение концентрации титаната свинца $(x)$ приводит к значительному росту температуры максимума $\varepsilon / \varepsilon_{0}\left(T_{m}\right)$ от $337 \mathrm{~K}$ (при $x=0.250$ ) до $447 \mathrm{~K}$ (при $x=0.400$ ) и подавлению релаксорных свойств. Аналогичный переход СЭР $\rightarrow$ нормальный сегнетоэлектрик наблюдался и при уменьшении концентрации бария $(z)$ в составе изучаемых ТР [17].

На рис. $1, b$ приведены зависимости $\varepsilon / \varepsilon_{0}(T)$, полученные при нагреве и охлаждении образцов с $x=0.250$, $0.325,0.350$ и 0.375 . Так же как и в случае изменения концентрации бария, переход СЭР $\rightarrow$ нормальный сегнетоэлектрик, наблюдаемый при увеличении концентрации $\mathrm{PbTiO}_{3}(x)$, сопровождается появлением и усилением температурного гистерезиса на кривых $\varepsilon / \varepsilon_{0}(T)$. Подобное поведение температурных зависимостей $\varepsilon / \varepsilon_{0}(T)$
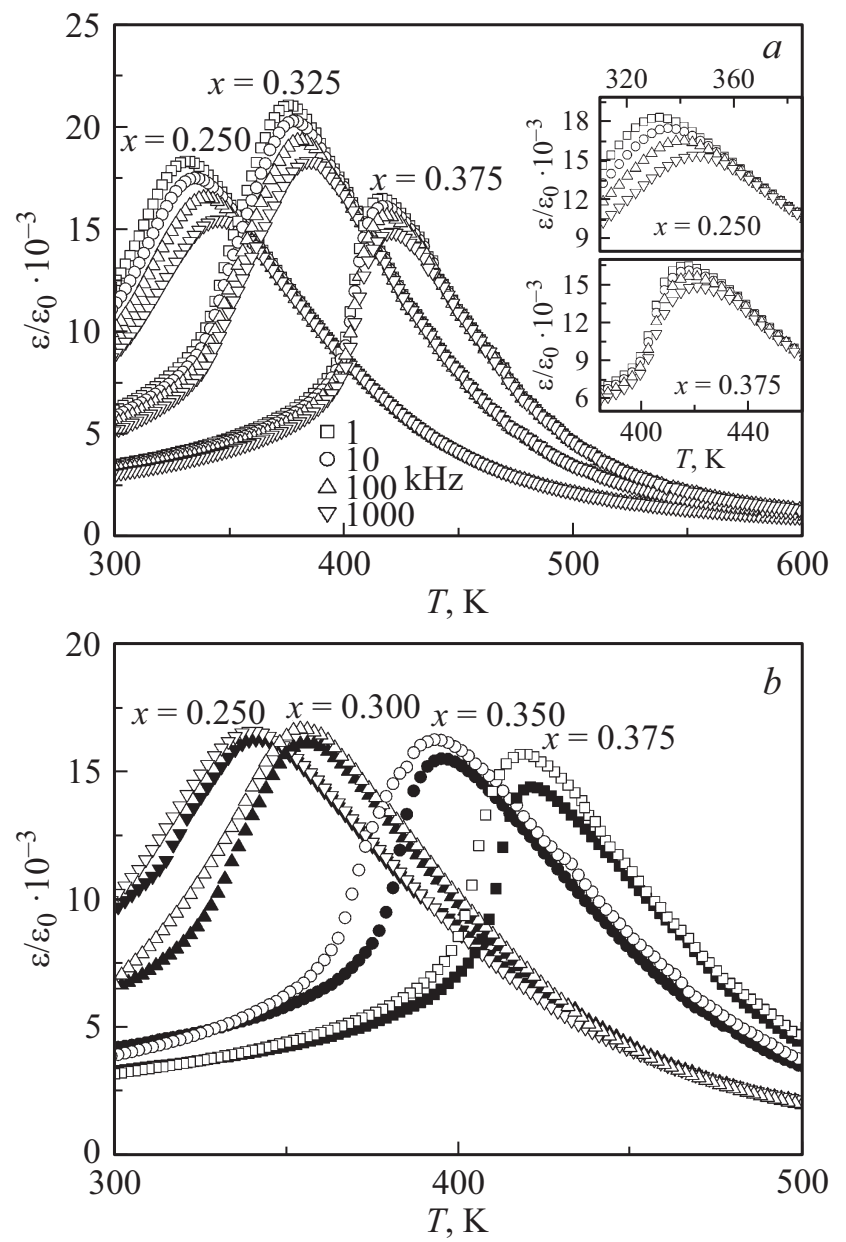

Рис. 1. (а) Зависимости $\varepsilon / \varepsilon_{0}(T)$ исследуемых ТР с различной концентрацией $\mathrm{PbTiO}_{3} \mathrm{c} x=0.250,0.325$ и 0.375 , полученные в режиме охлаждении при различных значениях $f$. $(b)$ Зависимости $\varepsilon / \varepsilon_{0}(T)$ исследуемых ТР с $x=0.250,0.300,0.350$ и 0.375 , полученные при $f=1 \mathrm{kHz}$ во время нагрева (заполненные символы) и охлаждения (пустые символы).

может быть следствием изменения характера ФП со второго рода (без температурного гистерезиса) на первый род (со значительным температурным гистерезисом). Согласно теории ФП второго рода Ландау, для термодинамических потенциалов не ниже шестой степени по параметру порядка на фазовой диаграмме линия переходов второго рода непрерывно переходит в линию ФП первого рода в критической точке ФП второго рода (трикритической точке) [19]. Таким образом, предполагается существование трикритической точки на $x$ - $T$ фазовой диаграмме изучаемых ТР при $x<0.325$. Вблизи трикритических точек на фазовых диаграммах можно ожидать усиления макроскопических откликов, связанных с величиной параметра порядка, в частности $\varepsilon / \varepsilon_{0}$. Например, возрастание значений $\left(\varepsilon / \varepsilon_{0}\right)_{m}\left(\varepsilon / \varepsilon_{0}\right.$ при $\left.T=T_{m}\right)$ наблюдалось вблизи трикритической точки на $x$ - $T$ фазовых диаграммах $\mathrm{TP}(1-x) \mathrm{Ba}\left(\mathrm{Zr}_{0.2} \mathrm{Ti}_{0.8}\right) \mathrm{O}_{3}-x\left(\mathrm{Ba}_{0.7} \mathrm{Ca}_{0.3}\right) \mathrm{TiO}_{3}[20]$ и 
Параметры диэлектрического отклика исследуемых твердых растворов

\begin{tabular}{c|c|c|c|c|c|c|c}
\hline$x$ & 0.250 & 0.275 & 0.300 & 0.325 & 0.350 & 0.375 & 0.400 \\
\hline$T_{\mathrm{VF}}, \mathrm{K}$ & 307 & 323 & 333 & 360 & 376 & 410 & - \\
$E_{a}, \mathrm{eV}$ & 0.0183 & 0.0180 & 0.0121 & 0.0106 & 0.0092 & 0.0053 & - \\
$T_{m}, \mathrm{~K}$ & 337 & 347 & 353 & 377 & 389 & 417 & 447 \\
$T_{d}, \mathrm{~K}$ & 530 & 536 & 540 & 549 & 550 & 555 & 557
\end{tabular}

$\mathrm{Ba}\left(\mathrm{Ti}_{1-x} \mathrm{Sn}_{x}\right) \mathrm{O}_{3}$ [21]. Локальные максимумы $\left(\varepsilon / \varepsilon_{0}\right)_{m}, \mathrm{a}$ также дисперсии $\varepsilon / \varepsilon_{0}$ и температурного сдвига $T_{m}$, наблюдались нами ранее на соответствующих зависимостях от концентрации бария в исследуемых ТР вблизи предполагаемой трикритической точки $(z \approx 0.125)$ [17]. Однако в случае изучаемой керамики с различным содержанием $\mathrm{PbTiO}_{3}$, точное положение предполагаемой трикритической точки на фазовой диаграмме определить затруднительно из-за значительного концентрационного шага $x$

На основании результатов диэлектрической спектроскопии исследуемых керамических образцов с вариацией концентрации титаната свинца и бария нами были определены три характерные температуры: $T_{d}, T_{m}$ и $T_{\mathrm{VF}}$. Величина $T_{d}$ определялась по отклонению кривой обратной $\varepsilon / \varepsilon_{0}$ от выполнения линейного закона Кюри-Вейса в параэлектрической области. $T_{\mathrm{VF}}$ вычисляли из соотношения Фогеля-Фулчера: $f=f_{0} \exp \left[-E_{a} / k\left(T_{m}-T_{\mathrm{VF}}\right)\right]$, где $f_{0}$ - частота попыток преодоления потенциального барьера (в нашем примере $f_{0} \sim 10^{11}$ ); $E_{a}$ - энергия активации; $k$ - постоянная Больцмана.

В таблице приведены основные параметры диэлектрического отклика изучаемых ТР с различной концентрацией титаната свинца $(x)$. Величины $E_{a}$ TP с ярко выраженными свойствами СЭР находятся в диапазоне $0.02-0.01 \mathrm{eV}$, что сопоставимо со значениями, зафиксированными в близких по химическому составу TP $0.75 \mathrm{PMN}-0.25 \mathrm{PT} \quad\left(E_{a} \approx 0.017 \mathrm{eV}\right.$ [22]), а также в $\mathrm{C} Э \mathrm{P} \mathrm{PbSc}_{1 / 2} \mathrm{Nb}_{1 / 2} \mathrm{O}_{3}\left(E_{a} \approx 0.014 \mathrm{eV}\right.$ [23]) со спонтанным переходом в сегнетоэлектрическое состояние. При увеличении концентрации $x$ и при переходе в нормальное сегнетоэлектрическое состояние наблюдается значительное понижение $E_{a} \mathrm{TP}$, а величина $T_{\mathrm{VF}}$ по своим значениям приближается к $T_{m}$.

На рис. 2 представлены $x-T(a)$ и $z-T(b)$ фазовые диаграммы, полученные на основании экспериментальных результатов. Видно, что как при уменьшении концентрации $\mathrm{PbTiO}_{3}(x)$, так и при увеличении концентрации ионов $\mathrm{Ba}^{2+}(z)$ происходит однотипная последовательность трансформаций диэлектрических свойств в исследуемых ТР: медленное $(\sim 5 \%)$ уменьшение величины $T_{d}$ и более резкий $(\sim 30 \%)$ спад зависимостей $T_{\mathrm{VF}}$ и $T_{m}$ от концентраций $x$ и $z$. Как сказано ранее, при $T \sim T_{d}$ происходит переход из параэлектрического (PE) в эргодическое релаксорное состояние (ER). Дальнейшее охлаждение приводит к переходу в неэргодическое релаксор- ное (NER) или в сегнетоэлектрическое состояние (FE) в зависимости от выполнения закона Фогеля-Фулчера (в FE-состоянии $T_{\mathrm{VF}} \approx T_{m}$ ). Стоит отметить, что положение обозначенной на фазовой диаграмме границы между NER и FE-состояниями согласуется с оценкой степени размытия сегнетоэлектрического ФП, проведенной нами ранее для образцов с различной концентрацией бария [17]. Важной особенностью рассматриваемых фазовых диаграмм является факт расширения области стабильности ER-состояния при уменьшении концентрации $x$ или увеличении концентрации $z$. Аналогичная по виду фазовая диаграмма была построена авторами [24] при изучении ТP $(1-x) \mathrm{BaTiO}_{3}-x \mathrm{BaSnO}_{3}$, претерпевающих переход из FE в NER состояние при $x \approx 0.19$.

Несмотря на очевидное сходство поведения диэлектрических откликов изучаемых ТР при увеличении концентрации $x$ и при уменьшении концентрации $z$ (или наоборот), кристаллохимические мотивы возникновения релаксорного состояния и перехода в нормальное сегне-
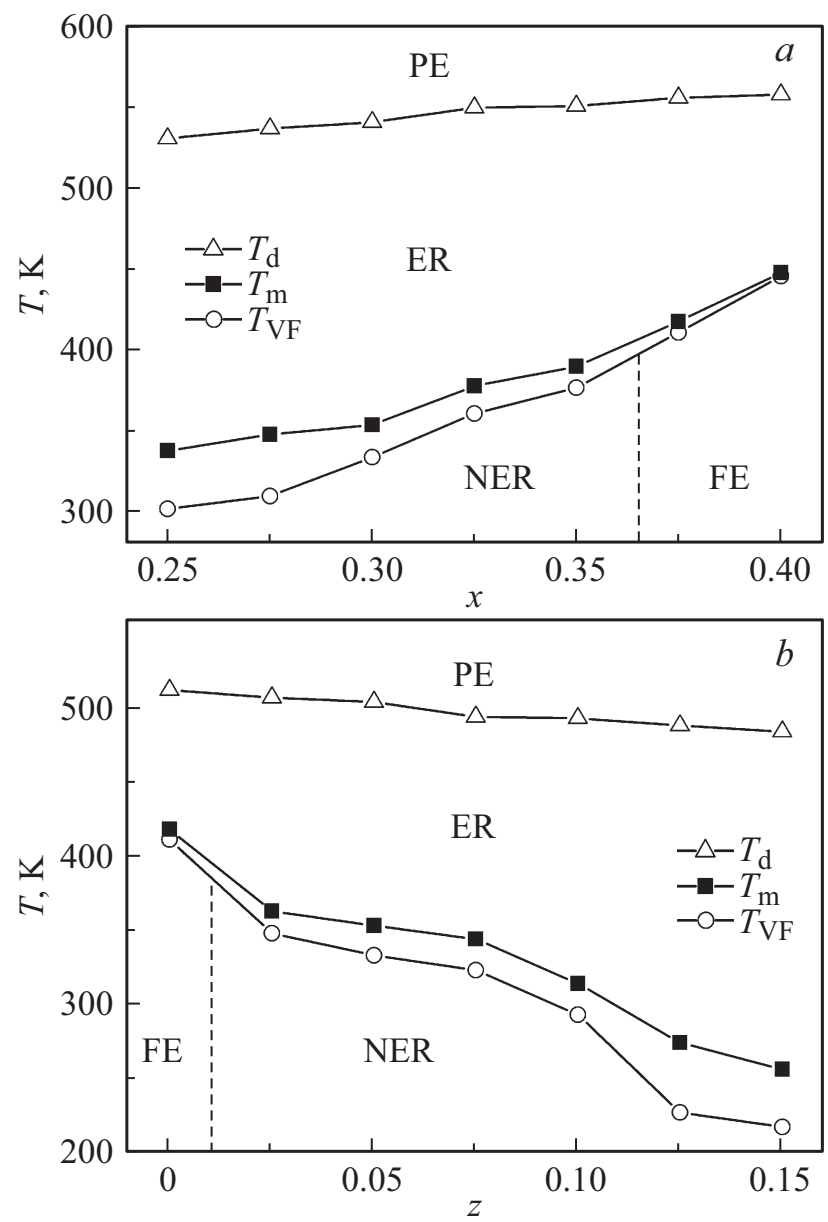

Рис. 2. Фазовые диаграммы исследуемых ТР, построенные на основе анализа диэлектрических спектров образцов с различными концентрациями $\mathrm{PbTiO}_{3}(a)$ и бария $(b)$. $\mathrm{PE}$ - параэлектрическое, ER - эргодическое релаксорное, NER - неэргодическое релаксорное, $\mathrm{FE}-$ сегнетоэлектрическое состояние. 
тоэлектричское состояние различаются. Считается, что неупорядоченное распределение разновалентных ионов $\mathrm{Mg}^{2+}, \mathrm{Zn}^{2+}, \mathrm{Ni}^{2+}$ и $\mathrm{Nb}^{5+}$ в $B$-подрешетке структуры перовскита является причиной возникновения случайных электрических полей и, соответственно, проявления свойств СЭР [10]. Замещение комплекса $\left[B_{1 / 3}^{2+} \mathrm{Nb}_{2 / 3}^{5+}\right]^{4+}$ ионами $\mathrm{Ti}^{4+}$ способствует уменьшению композиционного беспорядка в системе, подавлению случайных электрических полей и, как следствие, переходу в нормальное сегнетоэлектрическое состояние. Усиление релаксорных свойств, при изовалентном замещении в $A$-подрешетке ионов $\mathrm{Pb}^{2+}$ на ионы $\mathrm{Ba}^{2+}$, возможно за счет искажения октаэдров $\mathrm{BO}_{6}$ вблизи атомов бария с большим ионным радиусом в сравнении с $\mathrm{Pb}^{2+}[25,26]$. В результате возникают локальные упругие поля, которые в случае неупорядоченного распределения ионов в $A$-подрешетке могут выступать в качестве аналога случайных электрических полей, способствуя подавлению дальнего сегнетоэлектрического порядка. Кроме того, замещение стереоактивных ионов $\mathrm{Pb}^{2+}$ с неподеленной парой электронов на ионы $\mathrm{Ba}^{2+}$ со сферическим (изотропным) распределением электронной плотности приводит к снижению степени направленности (степени ковалентности) химических $A-\mathrm{O}$ связей и, по мнению авторов [25], способствует появлению случайных электрических полей.

\section{4. Заключение}

В работе исследованы диэлектрические спектры образцов системы $\mathrm{Pb}_{(1-z)} \mathrm{Ba}_{z}\left(\mathrm{Mg}_{1 / 3} \mathrm{Nb}_{2 / 3}\right)_{m}\left(\mathrm{Zn}_{1 / 3} \mathrm{Nb}_{2 / 3}\right)_{y}$. $\left(\mathrm{Ni}_{1 / 3} \mathrm{Nb}_{2 / 3}\right)_{n} \mathrm{Ti}_{x} \mathrm{O}_{3}$ c различной концентрацией титаната свинца и бария. Обнаружено, что как при увеличении концентрации титаната свинца, так и при уменьшении концентрации бария происходит переход из СЭР в нормальное сегнетоэлектрическое состояние, который сопровождается появлением и усилением температурного гистерезиса на кривых $\varepsilon / \varepsilon_{0}(T)$. Сделано предположение о существовании трикритической точки на $x-T$ фазовой диаграмме изучаемых ТР при $x<0.325$. На основании анализа экспериментальных данных определены характерные температуры исследуемых $\mathrm{TP}\left(T_{d}, T_{m}\right.$ и $\left.T_{\mathrm{VF}}\right)$ и построены $x$-T и $z-T$ фазовые диаграммы с выделенными границами существования параэлектрического, эргодического и неэргодического релаксорного и сегнетоэлектрического состояний. Показано, что несмотря на различные кристаллохимические мотивы возникновения релаксорного состояния и перехода в нормальное сегнетоэлектричское состояние в исследуемых ТР с замещением в $A$ - и $B$-подрешетках, общие черты фазовых диаграмм сохраняются: медленное $(\sim 5 \%)$ уменьшение величины $T_{d}$ и более резкий $(\sim 30 \%)$ спад зависимостей $T_{\mathrm{VF}}$ и $T_{m}$, их разделение при уменьшении концентрации титаната свинца и увеличении концентрации бария.

\section{Список литературы}

[1] S.-E. Park, T.R. Shrout. J. Appl. Phys. 82, 1804 (1997).

[2] S. Zhang, F. Li. J. Appl. Phys. 111, 031301 (2012).

[3] E. Sun, W. Cao. Prog. Mater. Sci. 65, 124 (2014).

[4] G. Burns, F.H. Dacol. Phys. Rev. 28, 2527 (1983).

[5] D. Viehlandl, S.J. Jang, L.E. Cross. J. Appl. Phys. 68, 2916 (1990).

[6] A.A. Bokov, Z.-G. Ye. J. Mater. Sci. 41, 31 (2006).

[7] G.A. Samara. J. Phys.: Condens. Matter. 15, R367 (2003).

[8] J. Toulouse, R.K. Pattnaik. J. Phys. Chem. Solids 57, 1473 (1996).

[9] B. Dkhil, P. Gemeiner, A. Al-Barakaty, L. Bellaiche, E. Dul'kin, E. Mojaev, M. Roth. Phys. Rev. B 80, 064103 (2009).

[10] V. Westphal, W. Kleemann, M.D. Glinchuk. Phys. Rev. Lett. 68, 847 (1992).

[11] S. Tinte, B.P. Burton, E. Cockayne, U.V. Waghmare. Phys. Rev. Lett. 97, 137601 (2006).

[12] А.Я. Данцигер, О.Н. Разумовская, Л.А. Резниченко, В.П. Сахненко, А.Н. Клевцов, С.И. Дудкина, Л.А. Шилкина, Н.В. Дергунова, А.Н. Рыбянец. Многокомпонентные системы сегнетоэлектрических сложных оксидов: физика, кристаллохимия, технология. Аспекты дизайна пьезоэлектрических материалов. Новая книга, Ростов на/Д. (2001). T. $1,2.900 \mathrm{c}$.

[13] М.В. Таланов, Л.А. Шилкина, Л.А. Резниченко. ФТТ 54, 930 (2012).

[14] М.В. Таланов, О.Н. Разумовская, Л.А. Шилкина, Л.А. Резниченко. Неорган. материалы 49, 1027 (2013).

[15] M.V. Talanov, L.A. Shilkina, L.A. Reznichenko. Sens. Actuators A 217, 62 (2014).

[16] М.В. Таланов, О.А. Бунина, М.А. Бунин, И.Н. Захарченко, Л.А. Резниченко. ФТТ 55, 288 (2013).

[17] М.В. Таланов, С.П. Кубрин, А.А. Павелко, Л.А. Резниченко. ФТТ 58, 1124 (2016).

[18] L. Swarz, T.R. Shrout. Mater. Res. Bull. 17, 1245 (1982).

[19] Л.Д. Ландау, Е.М. Лифшиц. Статистическая физика. Наука, M. (1976). $583 \mathrm{c}$.

[20] A.W. Liu, X. Ren. Phys. Rev. Let. 103, 257602 (2009).

[21] J. Gao, Y. Wang, Y. Liu, X. Hu, X. Ke, L. Zhong, Y. He, Xi. Ren. Sci. Rep. 7, 40916 (2017).

[22] A.A. Bokov, Z.-G. Ye. Phys. Rev. B 65, 144113 (2002).

[23] F. Chu, I.M. Reaney, N. Setter. Ferroelectrics 151, 1-4, 343 (1994).

[24] C. Lei, A.A. Bokov, Z.-G. Ye. J. Appl. Phys. 101, 084105 (2007).

[25] E. Dul'kin, B. Mihailova, M. Gospodinov, M. Roth. J. Appl. Phys. 112, 064107 (2012).

[26] A. Rohrbeck, G. de la Flor, M.I. Aroyo, M. Gospodinov, U. Bismayer, B. Mihailova. J. Phys.: Condens. Matter. 28, 475902 (2016). 